Why incompatibilism about mental causation is incompatible with non-reductive physicalism

Authors' details:

Jonas Christensen

Department of Philosophy

Aarhus University

Jens Chr. Skous Vej 7

8000 Aarhus

Denmark

filjfc@cas.au.dk

\title{
Umut Baysan
}

Faculty of Philosophy

University of Oxford

56 Woodstock Road (St Anne's College)

OX2 6HS, Oxford, UK

umut.baysan@philosophy.ox.ac.uk 


\title{
Why incompatibilism about mental causation is incompatible with non-reductive physicalism
}

\begin{abstract}
The exclusion problem is meant to show that non-reductive physicalism leads to epiphenomenalism: if mental properties are not identical with physical properties, then they are not causally efficacious. Defenders of a difference-making account of causation suggest that the exclusion problem can be solved because mental properties can be difference-making causes of physical effects. Here, we focus on what we dub an incompatibilist implementation of this general strategy and argue against it from a non-reductive physicalist perspective. Specifically, we argue that incompatibilism undermines either the non-reductionist or the physicalist aspirations of non-reductive physicalism.
\end{abstract}

\section{Keywords:}

Mental Causation; Interventionism; Proportionality; Disjunctive Properties; Causal Closure; Physicalism

\section{Introduction}

According to difference-making accounts of causation, in cases of causation, the causally efficacious properties are those that make a difference as to whether or not the effect occurs. A significant motivation for such accounts is that they supposedly capture both scientific and non-scientific practice of identifying causes. Nowhere is this more apparent than in the literature on higher-level causation, where proponents of difference-making accounts of 
causation - call them DM theorists - have argued that their account saves the causal efficacy of mental and other higher-level properties from the exclusion problem, namely the problem that mental and other higher-level properties appear to be causally excluded by physical properties. (Here, we are primarily interested in mental properties and mental causation, but everything we say can be extended to cover other higher-level properties that are invoked in the special sciences and higher-level causation). There are different ways to cash out the general idea that causes are those that make a difference to their effects. Accordingly, there are also different ways to implement the general strategy of treating causes as differencemakers in order to solve the exclusion problem. We identify two strands in the literature $-\mathrm{a}$ compatibilist and an incompatibilist approach, respectively - and target the incompatibilist implementation on the grounds that it offers an unattractive solution to the exclusion problem from a non-reductive physicalist perspective.

In $\S 2$ we present the exclusion problem for non-reductive physicalism. In $\S 3$, we present how the exclusion problem can be addressed by DM theorists, distinguish between compatibilist and incompatibilist versions. The main argumentative parts of the paper are $\S 4$ and $\S 5$, where we present two problems for incompatibilist solutions. In $\S 4$ we argue that the theory of causation that backs up a prominent version of incompatibilism leads to a commitment to disjunctive properties. This commitment, in turn, puts pressure on the non-reductionist component of non-reductive physicalism. In $\$ 5$ we argue that insofar as incompatibilists can answer this objection, a more general problem arises for incompatibilism and its aspirations of providing a solution to the problem of mental causation that is acceptable from a physicalist viewpoint. The result is that incompatibilism leads to either reductive physicalism or non-physicalism. Either way, incompatibilism is incompatible with non-reductive physicalism. Of course, incompatibilism cannot lead to both reductive physicalism and nonphysicalism; in this sense, the two problems we raise are mutually exclusive. We will remain 
neutral as to which of these paths the incompatibilist must travel. Our conclusion will be that the incompatibilist solution to the exclusion problem is unavailable to the non-reductive physicalist, and it suffices for this conclusion that the incompatibilist is forced down either one of them.

\section{Non-reductive physicalism and the exclusion argument}

Jaegwon Kim $(1998 ; 2005)$ has famously argued that non-reductive physicalism about the mind (the variety of physicalism according to which mental properties are not identical with physical properties) leads to mental epiphenomenalism. To illustrate Kim's argument, consider the following putative case of mental causation:

(The Jones case) Jones believes that there is beer in the fridge. His belief - call this belief property $M$ - causes him to open the fridge door - call this behavioural physical effect $B .^{1}$

In line with standard non-reductive physicalist commitments, we make the following two assumptions about the Jones case. First, $M$ must have a physical base - presumably some neural property - which realizes $M$ and upon which $M$ supervenes. Call this property $N$. Second, $M$ could have been realized by a different physical neural property $N^{*}$. The former follows from a general physicalist commitment that mental properties are physically realized by, and thereby supervene on, physical properties. The latter follows from what we take to be the main motivation for non-reductionism about the mind, namely that mental properties are

\footnotetext{
${ }^{1}$ We borrow this example from Raatikainen (2010). While we talk of properties causing effects throughout, this may be taken as shorthand for saying that events, tropes, objects or entities from some other category cause effects in virtue of their properties. If a property is a cause of an effect in this sense, we say that it is causally efficacious.
} 
multiply realizable by distinct physical properties (Putnam 1967). Co-extension of properties $F$ and $G$ is at least a necessary condition for identifying $F$ with $G$; and since $M$ is multiply realizable by different physical properties $N$ and $N^{*}$, it fails to be co-extensive with $N$.

Now, assume the principle of the causal closure of the physical (henceforth 'Closure'):

(Closure) If a physical event $e$ has a cause at time $t$, then $e$ has a sufficient physical cause at $t$.

If Closure is true, in the Jones case, $B$ must have a physical sufficient cause, presumably the neural property $N$ upon which $M$ supervenes and which is co-instantiated with $M$ at some time $t$. Now, if $N$ is a sufficient cause of $B$ at $t$, how can $M$ be a cause of $B$ at $t$ ? There are two options. Either $M$ is identical with $N$ (so $M$ is a cause of $B$ at $t$ because $N$ is cause of $B$ at $t$ ) or $M$ and $N$ are not identical, in which case $M$ and $N$ are overdetermining causes of $B$ at $t$. According to Kim, neither option is available to non-reductive physicalism. On the one hand, identifying $M$ with $N$ undermines the non-reductionist part of non-reductive physicalism. On the other hand, treating $M$ as an overdetermining cause of $B$ runs afoul of what Kim dubs the exclusion principle (henceforth 'Exclusion'):

(Exclusion) No effect has more than one (simultaneous) sufficient cause unless it is a case of genuine causal overdetermination, and causation by mental properties is not genuine causal overdetermination. ${ }^{2}$

\footnotetext{
${ }^{2}$ See Kim $(2005,42)$. Note that Kim's version of the exclusion principle contains only the first conjunct of Exclusion. He adds the claim that mental (and other supervenient) properties are not overdetermining causes as a separate premise of his argument.
} 
Kim's conclusion is that if non-reductive physicalism is true, then $M$ is excluded from causing $B$ in the Jones case; and more generally, mental properties are excluded from causing physical effects. Understandably, many (including Kim himself) find this epiphenomenalist conclusion unpalatable. Hence, the exclusion argument is typically taken to present an exclusion problem for non-reductive physicalists. We will assume throughout that mental epiphenomenalism is not an option for non-reductive physicalists, and that they must reject one of the premises that leads to this problem.

\subsection{Reduction and the disjunctive move}

Kim's preferred solution to the exclusion problem is to give up the assumption that mental properties are irreducible to physical properties. So, for Kim, the exclusion argument is a reductio against the non-reductionist component of non-reductive physicalism. This means that Kim must respond to the argument from multiple realizability against reduction. We will not consider his strategy for doing so here (but see Kim 1992). Instead we wish to briefly flag a reductionist strategy known as the disjunctive move which is meant to accommodate the multiple realizability of mental properties. Recall that multiple realizability prevents the identification of a mental property with any of its specific physical realizers. For example, in the Jones case, $M$ is not identical with $N$ since $M$ could have been realized by $N^{*}$. In saying this, however, it is not ruled out that there is some physical property other than $N\left(\right.$ or $\left.N^{*}\right)$ with which $M$ could be identified. According to the disjunctive move, there $i s$ such a property, namely the disjunctive property which consists of the disjunction of all of $M$ 's (actual and possible) realizers. Call this disjunctive property $D P$. Unlike any of $M$ 's specific physical realizers, $D P$ is (necessarily) co-extensive with $M$. Hence, the main obstacle to reducing $M$ to a physical property - namely $D P$ - is removed. 
Opponents of the disjunctive move typically question the move on the grounds that disjunctive properties in general are metaphysically or scientifically illegitimate (see Fodor 1974; Armstrong 1989; Heil 1992; Kim 1992; Audi 2014). ${ }^{3}$ We will not adjudicate this issue here but simply point out that the dispute over the viability of the disjunctive move is not over the viability of the reduction proposed by the move as such; instead, it is a dispute about the existence of disjunctive properties. In other words, it is agreed that if disjunctive properties such as DP exist, the path to psycho-physical reduction would be cleared of the argument from multiple realization since $M$ and $D P$ are (at least) nomologically co-extensive. The contentious issue is the truth of the antecedent (see also Clapp 2001, 111-112 for this point). This will be important later as we will argue in $\S 4$ that the incompatibilist solution that we will focus on is based on an account of causation which requires the existence of disjunctive properties. ${ }^{4}$ Hence, as we shall argue, under this theory of causation, nonreductive physicalists' response to the disjunctive move is undercut.

\footnotetext{
${ }^{3}$ An anonymous referee has suggested a different line of resistance to the disjunctive move: Accept that $D P$ is a legitimate property but hold that $M$ cannot be reduced to $D P$ since $M$ is singular and $D P$ is a plurality. Why think that $M$ is singular? One reason would be if $M$ fails to be necessarily coextensive with any legitimate property that is a plurality. But if disjunctive properties are legitimate properties, there $i s$ such a property, namely $D P$. So, this objection to the disjunctive move would require other reasons for thinking that $M$ is singular. We do not see what they would be, and, in any case, we provide independent reasons for thinking that the reduction of $M$ to $D P$ is compelling if disjunctive properties are legitimate in $§ 4.1$.

${ }^{4}$ More accurately, we will argue that the account requires disjunctive causes. Since we take the relata of causation to be properties, the account therefore requires disjunctive properties.
} 


\section{Compatibilist and incompatibilist solutions to the exclusion problem}

In its most general form, the difference-making account of causation simply says that causes are those that make a difference to their effects. Using this idea, many have argued that the epiphenomenalist conclusion to the exclusion argument should be rejected by non-reductive physicalists, since mental properties are sometimes difference-makers for, and thereby causes of, physical effects. Of course, rejecting the conclusion of an argument requires rejecting one of its premises. Which one? Here DM theorists disagree. This disagreement amongst DM theorists over which premise of the exclusion argument to reject maps onto a disagreement over how best to construe the relevant notion of difference-making. Before giving some examples of different models, let us prefigure the discussion by noting that on some construals of difference-making, Exclusion turns out false. That is, in normal cases of mental causation, the relevant physical effect is caused by some physical property and sufficiently caused by an (irreducible) mental property. We shall call this a compatibilist solution to the exclusion problem (or compatibilism) since it holds that mental causation takes place alongside, and hence is compatible with, simultaneous and distinct underlying physical causation. $^{5}$

On other construals of difference-making, Closure turns out false. That is, in normal cases of mental causation, there is no underlying physical cause of the relevant physical behavioural effect. We call this an incompatibilist solution to the exclusion problem (or incompatibilism) since it holds that, in general at least, causation of a physical effect $e$ via a mental property is incompatible with causation of $e$ via a distinct physical property.

\footnotetext{
${ }^{5}$ We borrow this terminology from Horgan $(1997,166)$.
} 
While our target in what follows is incompatibilism, (for illustrative purposes) let us quickly give a prominent example of a current compatibilist view, namely Woodward's interventionist theory of causation. ${ }^{6}$ As the name suggests, the notion of an intervention plays a key role in the theory. Roughly, an intervention is a (potentially counterfactual) causal manipulation of the value taken by some property (e.g., a manipulation that changes the property from being instantiated to not being instantiated or vice versa). Ignoring some complexities irrelevant to the present discussion, according to Woodward's interventionism, the truth-conditions for difference-making are as follows:

$F$ is a difference-maker for (and therefore a cause of) $G$ if and only if there is a possible intervention on $F$ (i.e., a causal manipulation of $F$ 's value) that will change the value of $G$. (More colloquially, $F$ is a cause of $G$ when it is possible to manipulate $G$ via a manipulation of $F$.)

Applying the interventionist understanding of difference-making to the Jones case, we can see that both $M$ and $N$ come out as causes of $B$. First, there is a possible intervention which changes the value of $M$ from being present to being absent and under which the value of $B$ also changes from occurring to non-occurring. For instance, if someone had informed Jones that there is no beer in the fridge (thereby causing $M$ to be absent), Jones would not have opened the fridge ( $B$ would not have occurred). (Likewise, if Jones had acquired the belief that there is beer in the fridge by way of intervention, he would have opened the fridge). Second, there is a possible intervention which changes the value of $N$ from being present to

\footnotetext{
${ }^{6}$ Note that the following is based on Woodward's interventionist account as presented in Woodward (2015), rather than his original account in Woodward (2003). It is a controversial issue whether the latter allowed for the theory to be applied to models involving properties that stand a in a non-causal dependence relation (e.g. realization).
} 
being absent and under which the value of $B$ also changes from occurring to not-occurring. For instance, informing Jones that the fridge contains no beer would also change the value of $N$ from being present to being absent (since his belief that there is beer in the fridge supervenes on $N$ ). (Likewise, if an intervention were to cause Jones to instantiate $N$, he would open the fridge since $N$ necessitates $M$ and the presence of $M$ leads to opening the fridge). So, by interventionist standards, $M$ and $N$ are both causes of $B$. Assuming $N$ is a sufficient cause of $B$ (as per Closure) and that the Jones case is a normal case of mental causation, Exclusion is therefore false. ${ }^{7}$

The above is a highly condensed version of the interventionist response to the exclusion problem. We present it merely as an illustrative example of a compatibilist strategy and won't assess the virtues of the theory or compatibilism more generally here. Instead, our focus will be exclusively on incompatibilist strategies. A prominent example of such a strategy is articulated in the works of Peter Menzies and Christian List. ${ }^{8}$ Menzies and List's treatment of the exclusion problem employs a theory of proportional causation (List and Menzies 2009; Menzies and List 2010; Menzies 2008; 2013). ${ }^{9}$ Like Woodward, Menzies and List explicitly

\footnotetext{
${ }^{7}$ Woodward is explicit that his account of causation shows that the exclusion principle is false. See Woodward $(2015,344)$.

${ }^{8}$ But see also Yablo (1992) and (1997), and Raatikainen (2010).

${ }^{9}$ Although Menzies and List agree with many features of Woodward's interventionism and at one point explicitly appeal to 'a simplified version of an "interventionist" theory advanced by James Woodward' (Menzies and List, 2010, 108), as will be clear in what follows, the conditions under which a property is a cause according to Menzies and List are importantly different from Woodward's theory as we considered above. Here, we reserve the term 'interventionism' for the view that is defended by Woodward.
} 
take causes to be those that make a difference to whether or not an effect occurs. They provide the following truth conditions for difference-making:

The presence of $F$ makes a difference to the presence of $G$ in the actual situation just in case (i) if any relevantly similar possible situation instantiates $F$, it instantiates $G$; and (ii) if any relevantly similar possible situation instantiates not- $F$, it instantiates not-G. (List and Menzies 2009, 482). ${ }^{10}$

Adopting a term coined by Yablo (1992), when these two conditions are met, Menzies and List say that $F$ is proportional to $G$ (or a proportional cause of $G$ ). We label this theory of causation proportionalism.

Like interventionism, proportionalism judges that Jones's belief is a cause of him opening the fridge. In all relevantly similar situations, Jones will open his fridge when he believes his fridge contains beer and will not do so when he does not have this belief. So, $M$ is a proportional cause of $B$. What about $M$ 's physical realizer $N$ ? Since $M$ is multiply realizable, there are relevantly similar situations where $N$ is absent but $B$ nevertheless takes place. These are situations where $N$ is absent but a different physical realizer of $M$, say $N^{*}$, is present. In those situations, $M$ is also present (because $N^{*}$ realizes $M$ ) so Jones opens his fridge. $N$ therefore fails to be a cause of $B$ in the Jones case since it fails to be proportional to this effect (see List and Menzies 2009, 495. Although the details of the views differ in various ways,

\footnotetext{
${ }^{10}$ Menzies and List go on to cash out the notion of relevantly similar situations in terms of nearby possible worlds and offer their own modal semantics which differ in important ways from a standard Lewisian treatment of counterfactuals. Here we stick to Menzies and List's less formal definition of difference-making. Everything we say involving 'relevantly similar situations' could be rephrased in terms of nearby possible worlds using Menzies and List's modal semantics.
} 
Yablo and Raatikainen arrive at the same result using similar considerations. See Yablo 1992, 278 and Raatikainen 2010, 358). ${ }^{11}$ This result captures the idea that difference-makers should not be overly specific $-N$ fails as a cause because it comes with irrelevant causal information not required to bring about $B$ (Yablo 1992, 276; List and Menzies 2009, 480). More schematically, under proportionalism, a variable $V$ taking the value of being present fails to cause the occurrence of $E$ if, in any relevantly similar situation where $V$ takes the value of being absent, $E$ nevertheless occurs. Properly generalized, the proportionalist treatment of the Jones case shows that, in ordinary cases of mental causation, mental properties are difference-makers (and hence causes) at the expense of their physical realizers---a phenomenon Menzies and List dub 'downward exclusion' since the higher-level property

\footnotetext{
${ }^{11}$ When presenting the interventionist response to the exclusion problem, we assumed that the intervention on $N$ 'wipes out' $M$. In other words, the intervention is such that $N$ is not replaced by a different physical realizer, $N^{*}$ (in which case $M$ would still be present). But what motivates this assumption? Could an intervention on $M$ not be such that $N$ is replaced by $N^{*}$ in which case $B$ would occur nonetheless? And if so, would interventionism then not support an incompatibilist solution to the exclusion problem? We think not. First, it is important to note that interventionism does not require that for $N$ to cause $B$, every possible intervention on $N$ leads to change in the value of $B$. All interventionism requires is that there is at least one such possible intervention. And there is; namely an intervention that removes $N$ without replacing it with a different realizer of $M$. There is no assumption that this intervention is more appropriate or relevant than an intervention which replaces $N$ with $N^{*}$, all that is assumed is that it is possible. Now, for interventionism to yield the result that $N$ fails to cause $B$, it would have to be the case that there is no possible intervention on $N$ which would lead to a change in the value of $B$. As Woodward says, however, this would require that 'some kind of back-up mechanism were in place that ensures that when $[N]$ does not occur, an alternative realizer of $[M]$ does' (Woodward 2015, fn. 1), which, in general at least, seems implausible. We thank an anonymous referee for prompting us to consider this.
} 
excludes the lower-level property from being a difference-making cause. This means that, in cases of mental causation, Closure fails (for explicit mention of this, see Menzies 2008, 216 and Raatikainen 2010, 361).

Does incompatibilism provide an attractive solution to the exclusion problem? One strategy would be to assess if the theory of causation that backs up this solution is an attractive one. Such a 'causation-first approach' is largely the backdrop against which the current debate on the difference-making solutions to the exclusion problem are assessed (see McDonnell 2016). ${ }^{12}$ Here we wish to approach the dispute in a different manner and assess if this solution is attractive from a non-reductive physicalist point of view. Our approach is motivated by the fact that incompatibilism is presented as a solution to the exclusion problem, and the exclusion problem is raised against non-reductive physicalism. Hence, it is reasonable to assess how well this solution fits with standard non-reductive physicalist commitments. As we will go on to argue, the incompatibilist solution proposed by Menzies and List faces two problems in this respect. First, the proportionalist account of causation that Menzies and List appeal to pushes the incompatibilist towards a form of psycho-physical reduction. Second, it is questionable whether rejecting Closure is compatible with physicalism. We present these two problems in $\S 4$ and $\S 5$ respectively.

\section{Proportionalism and disjunctive causes}

Proportionalists often illustrate their take on mental causation by using examples involving properties standing in a determinable-determinate relation (e.g., List and Menzies 2009, 4802 and Zhong 2014, 353-4; the following example originates from Yablo 1992). Assume that Sophie, a pigeon, is trained to peck whenever she is presented a red object. On a particular

\footnotetext{
${ }^{12}$ The term 'causation-first approach' is also due to McDonnell.
} 
occasion, she is presented a scarlet object and she pecks. Which property caused the pecking - the determinable property red or its subvenient determinate property scarlet? According to proportionalists, red satisfies both conditions for causation - in all relevantly similar situations in which Sophie is presented a red object, she pecks; in all relevantly similar situations in which she is presented a non-red object, she does not peck. Scarlet, on the other hand, fails the second condition - there are relevantly similar situations where Sophie is presented a non-scarlet object but where she pecks regardless. These are situations where she is presented objects that have a different shade of red, say crimson. So, while the object presented was not crimson, it might have been, in which case Sophie would have pecked regardless. In other words, scarlet is too specific to make a difference to the pecking since other values (e.g., crimson) also correlates with this effect. The case is instructive because the determinate property scarlet fails to cause Sophie's pecking for the same reason that $N$ fails to cause $B$ in the Jones case - there are relevantly similar situations (e.g., one where the object presented to Sophie is crimson) where scarlet takes the value of being absent and where Sophie pecks nonetheless.

It is easy enough, however, to imagine cases where red fails to cause the pecking by the same reasoning - as do Shapiro and Sober (2012). ${ }^{13}$ Say that a pigeon, call her Lucy, is trained to peck only at objects that are either red or blue. At some particular time, Lucy is presented a red chip. While the chip was not actually blue, it might have been. So, consider all relevantly similar counterfactual situations where the object presented is some colour which is not red. Some of these will be situations where the chip is blue and where Lucy pecks. Red therefore

\footnotetext{
${ }^{13}$ Note that Shapiro and Sober's example to make this point is different from the one we shall give. See also Weslake (2013) and Franklin-Hall (2016) for similar points in the context of proportionalism about causal explanation.
} 
fails the second condition for proportionality and fails to cause the pecking. Once again, the reasoning employed to reach this conclusion in the Lucy case is the same reasoning both according to which $N$ fails to cause $B$ in the Jones case and scarlet fails to cause the pecking in the Sophie case. What is common in all cases is that the target variable takes a different value across relevantly similar situations some of which are situations where the target effect nevertheless occurs. Hence, neither property being present is causing the effect according to proportionalism. This is not a special case. Ordinarily, multiple values of a putative cause variable may correlate with the same effect. Doing too much exercise and none at all are both correlated with a decreased life expectancy; Julie may enjoy the taste of vanilla and strawberry ice cream but dislike other flavours; so on and so forth. By parity of reasoning, failure to exercise does not cause premature death since doing too much would lead to the same result; the ice cream that Julie is eating being vanilla ice cream is not causing her enjoyment since had it been strawberry ice cream, she would have enjoyed herself nonetheless, etc.

Shapiro and Sober (2012) take these to be counterintuitive results and a strike against the proportionalist's ability to capture common-sense causal talk. We do not find intuitions so clear-cut in these cases to be decisive against proportionalism per se, and in any case, we are not interested in the viability of proportionalism as a theory of causation in general. We are only interested in whether or not the solution to the exclusion problem that it supports is available to non-reductive physicalists. In the Jones and Sophie cases, there are obvious candidate properties that satisfy the proportionalist conditions for causation ( $M$ and red respectively). But which property is causally efficacious in the Lucy case? What the proportionalist needs is a property that is co-extensive with a disjunction of the values that correlate with Lucy pecking across relevantly similar situations (i.e., red and blue). No other property will do as no other property would be such that it is proportional to the pecking (i.e., 
such that its presence/absence correlates with the presence/absence of the pecking). The only candidate property we can think of that satisfies this requirement is the disjunctive property being red or blue. After all, there is nothing more to the values that correlate with Lucy pecking than the fact that they are either red or blue. ${ }^{14}$ If this is so, the proportionalist theory of causation requires the existence of disjunctive properties. We can state the forgoing argument in the following way:

(1) There are Lucy style cases, and in a Lucy style case, there is a property $P$ which is causally efficacious with respect to the relevant effect $E$.

(2) If proportionalism is true, $P$ is proportional to $E$.

(3) The only property proportional to $E$ in a Lucy style case is a disjunctive property.

(4) Therefore, proportionalism requires the existence of disjunctive properties.

Can this argument be resisted? We take (1) to be unproblematic. There are people who like some flavours of ice cream and not others, and likewise, there might be pigeons like Lucy. Furthermore, it would be absurd to hold that the relevant effects in such cases (Lucy pecking, Julie's enjoyment, etc.) are uncaused. Note also that (2) is a consequence of (1) given the proportionalist definition of causal efficacy as proportionality. ${ }^{15}$

\footnotetext{
${ }^{14}$ See Penczek (1997) for a similar argument.

${ }^{15}$ An anonymous referee has asked whether proportionalists might reject that $P$ causes Lucy's pecking despite being proportional to this effect on the grounds that $P$ isn't a natural kind property. In other words, proportionality isn't strictly sufficient for causation, it is also required that the property proportional to the effect is a natural kind. This response, however, does not help the proportionalist as it still leaves her with no causally efficacious property in the Lucy case (since the only property
} 
Understandably, (3) is the more controversial premise, and we anticipate two objections. The first objection goes as follows. The relation between, on the one hand, the cause of Lucy's pecking and on the other, red and blue, should not be construed as a disjunctive/disjunct relation but rather some other relation, e.g., functional realization. So, in the Lucy case, the causally efficacious property $P$ is not a disjunctive property with red and blue as its disjunct but rather a functional property with red and blue each functionally realizing $P$. If this objection is correct, then proportionalists are not committed to the existence of disjunctive properties.

We are not persuaded by this objection, for we do not believe construing the cause of Lucy's pecking as a functional property at the expense of either red or blue is an option for proportionalists given how functional realization is typically characterised in the literature (e.g. Block 1980 and McLaughlin 2006). We expand on this in $\$ 5$ but in short, functional realization requires that the realizing property performs the causal role associated with the relevant functional property. But according to proportionalism neither red nor blue performs the role of causing Lucy's pecking since neither satisfies the conditions for causation. So, neither red nor blue realizes a functional property associated with the causal role of causing Lucy's pecking. But perhaps there are non-standard construals of functional realization that the proportionalist could appeal to? Of course, we cannot rule out that there are non-standard

proportional to Lucy pecking is $P$ ). While we grant that there is nothing logically contradictory about denying that Lucy's pecking is uncaused, but this sounds to us empirically implausible. One can argue over which property is causing Lucy's pecking, but it does not seem to us that one can reasonably argue that there is no causally efficacious property at all. 
construals of functional realization which the proportionalist can appeal to but if so, we would want to be told what they are. Until then, we feel entitled to endorse (3).

The second objection targets our example. ${ }^{16}$ Perhaps situations where Lucy is presented with a blue chip fail to be relevantly similar to the actual situation. If so, red would come out as a cause of the pecking on the proportionalist account since every relevantly similar situation where Lucy is presented with a non-red chip are situations where the chip presented is neither red nor blue and the pecking doesn't occur. There is thus no need to posit the disjunctive property being red or blue as the cause of Lucy's pecking in the actual situation. In response, we note that this threatens to undermine the incompatibilist claim that, in Jones and Sophie cases, the mental/determinable property is causally efficacious at the expense of the realizer/determinate property. First, if Lucy being presented with a blue chip fails to be relevantly similar to the actual situation (where she is presented with a red chip), then situations where Sophie is presented with a crimson chip (or chips with other shades of red) would also fail to be relevantly similar to the actual situation (where she is presented with a scarlet chip). If so, crimson would cause Sophie's pecking in the actual situation since every relevantly similar situation where she is presented a non-crimson chip are situations where the chip presented is non-red and Sophie doesn't peck. However, if determinable causation and mental causation are analogous (as proportionalists claim), this would also undermine the claim that $N$ fails to cause $B$ in the Jones case. If crimson is efficacious in the Sophie case and $N$ is analogous to crimson in the Jones case, then $N$ is efficacious in the Jones case. Of course, it may be denied that mental causation and determinable causation are analogous after all. Perhaps crimson and red are both efficacious in the Sophie case (because the relevantly similar situations in which the chip presented is non-crimson include only those where the

\footnotetext{
${ }^{16}$ Thanks to an anonymous referee for raising this objection.
} 
chip is no other shade of red) while only $M($ and not $N$ ) is efficacious in the Jones case. But what would motivate such a claim? The notion of 'relevantly similar situations' would have to be construed in precisely such a manner that (in the Sophie case) the chip being scarlet fails to be relevantly similar to the actual situation in which the chip is crimson, whereas (in the Jones case) Jones having a slightly different realizer of $M$, say $N^{*}$, is relevantly similar to the actual situation in which has $N$. It remains to be seen whether there is a way to do this in a non-ad hoc and satisfactory manner. ${ }^{17}$

As we noted in $\S 2.1$, the metaphysical status of disjunctive properties is a controversial matter. We take no stand on this issue here. Our conclusion (so far) is simply this: if disjunctive properties are problematic, then so is proportionalism. For if there are no disjunctive properties then there are no proportional causes in the cases above; and if there are no proportional causes there are no causes simpliciter. Recall, however, that our aim is not to evaluate the plausibility of proportionalism, but rather to consider whether the position provides a solution to the exclusion problem from the perspective of non-reductive physicalism. We will now argue that the commitment to disjunctive properties undermines the non-reductionist component of the view.

\subsection{Proportionalism and the disjunctive move}

Let us return to cases of mental causation. As mentioned in $\S 2.1$, according to the disjunctive move, mental properties are reducible to (identifiable with) disjunctions of their physical

\footnotetext{
${ }^{17}$ Another option is to claim that determinable causation and mental causation are analogous and that in both cases, the realizer/determinate property causes the effect along with a distinct mental/determinable property. If this is the proportionalist claim, it would support a compatibilist solution to the exclusion and our arguments do not apply to it.
} 
realizers. The question now is this. If proportionalists are committed to the existence of (even only some) disjunctive properties as causes, then what are the motivations for rejecting the disjunctive move against the non-reductionist component of non-reductive physicalism? As we said, the standard objection to the disjunctive move rests on anti-realism about disjunctive properties. But we have argued that proportionalists must be realists about disjunctive properties, otherwise they are left without causes in Lucy style cases. So, the proportionalist cannot resist the disjunctive move on standard grounds.

Could one accept that, for any mental property $M$, the disjunction of $M$ 's physical realizers form a disjunctive property $D P$, and at the same time deny that $M$ reduces to $D P$ ? We find this view unmotivated on several fronts. First, arguments from multiple realizability do not apply to $M$ and $D P$ - while $M$ is multiply realized by each of $D P$ 's disjuncts, $M$ is not multiply realized by $D P$ and some other property; after all, $M$ and $D P$ are (necessarily) coextensive. Second, there are no causal differences to ground the distinctness of $M$ and $D P$ since $M$ and $D P$ have exactly the same causal powers according to proportionalism. The reason is that if one property satisfies the conditions for proportional causation, so does any property which is co-instantiated with it across all relevantly similar situations. And since $M$ and $D P$ are co-extensive, co-instantiation in all relevantly similar situations is guaranteed. Third, even if the irreducibility of $M(v i s-\grave{a}-v i s D P)$ is granted, the fact that $M$ is co-extensive with causally indistinguishable physical property $D P$ arguably undermines the causal autonomy of $M$ (and other mental properties). Securing the causal autonomy of mental properties, however, is an explicit goal of many non-reductive physicalists. Menzies and List, for instance, argue that mental properties are autonomous vis-à-vis physical properties in the sense that they have novel causal powers not possessed by physical properties (Menzies and List 2010, 108). And Wilson agrees that non-reductive physicalism requires causal autonomy of mental properties but holds that it suffices for this that mental properties have distinctive 
causal profiles, such as causal powers that form a proper subset of some physical properties (Wilson 2011, 129). But if $M$ is causally indistinguishable from physical property $D P, M$ has neither novel causal powers nor a distinctive causal profile relative to $D P . M$ therefore fails to be autonomous from $D P$ on either account of causal autonomy. So, not only are there no obvious reasons for holding that $M$ is irreducible to $D P$, it is doubtful that such irreducibility in itself is enough to give non-reductive physicalists what they want.

Could one accept that $M$ reduces to $D P$ but deny that $D P$ is a physical property? Whether or not the domain of physical properties is closed under disjunction is, we take it, ultimately a matter of stipulation. In this sense, it is ultimately a matter of stipulation whether the disjunctive move should be considered a reductive physicalist move. As with physicalism, however, what is not a matter of stipulation is how philosophers engaged in the debate treat the extension of the physical domain for purposes of formulating psycho-physical reduction. Here it is clear that the disjunctive move is considered a form of psycho-physical reduction (Heil 1992, 64; Jaworski 2002, 91; Skiles 2016, 481; Beebee 2017, 297). ${ }^{18}$

We take these considerations to support our claim that the theory of causation on which the incompatibilist solution to the exclusion problem is based threatens to undermine the nonreductionist component of non-reductive physicalism. The proportionality aspect of List and

\footnotetext{
${ }^{18}$ An anonymous referee raises the following point: Suppose disjunctive properties are admitted to our ontology and that $D P$ is a legitimate property. Can the non-reductionist argue that, despite necessary co-extension of $M$ and $D P, M$ does not reduce to $D P$ because $D P$, as a disjunctive property, does not enter in the nomic relations that $M$ enters? We don't think this would be a successful nonreductionist strategy. If $D P$ and $M$ are necessarily co-extensive, they are nomologically co-extensive. Therefore, if $D P$ does not enter into nomic relation $R, M$ does not enter in $R$ either. (This response follows Kim 1992.)
} 
Menzies's solution requires disjunctive properties; and if disjunctive properties are allowed in one's ontology, then all else being equal, non-reductive physicalism cannot be defended from the disjunctive move. Note that this is not a problem for the compatibilist solution. The understanding of difference-making that the compatibilist solution is based on -i.e., interventionism - does not require disjunctive properties in the cases we discussed in the previous section. So, from a non-reductionist point of view, the compatibilist solution is superior to the incompatibilist solution.

\section{Incompatibilism, closure and physicalism}

Recall that the incompatibilist solution rejects Closure. But is rejecting this principle compatible with physicalism? The answer depends, of course, on how physicalism is understood. Not only is 'physicalism' a philosophical term of art with no agreed upon definition, the term is also used differently in different philosophical contexts. Since our interest here is in non-reductive physicalism about the mind (and other higher-level properties), we will narrow the focus of the question accordingly. Is rejecting Closure compatible with how contemporary philosophers engaged in debates over physicalism about the mind characterize physicalism (either explicitly or implicitly)?

Traditionally, a popular approach has been to define physicalism in terms of supervenience, with the idea being (roughly) that physicalism about the mind is true if and only if mental properties supervene on physical properties (Horgan 1982; Lewis 1983; Kim 1984; Jackson 1998). This is also the characterization Menzies and List adopt (List and Menzies 2009, 475; see also Menzies 2013, 59). On such accounts, physicalism is silent on issues about causation and expresses merely a necessary covariance between different domains of properties, which is compatible with the failure of Closure. 
That said, while most agree that psycho-physical supervenience in some form is necessary for physicalism, the contemporary literature reveals reasons to doubt that it is sufficient since psycho-physical supervenience is arguably compatible with anti-physicalist views such as emergentism (see Horgan 1993). The standard response to this worry is to distinguish physicalism from emergentism by way of the modal strength of the supervenience relation. Emergentists, it is claimed, are committed only to nomological psycho-physical supervenience whereas physicalists require metaphysical psycho-physical supervenience (see van Cleve 1990; Noordhof 2003; 2010; Fazekas 2014). However, such strategies are undermined if properties have their causal powers necessarily (a possibility which is intuitively orthogonal to the issue of whether physicalism is true), in which case the distinction between nomological and metaphysical supervenience collapses (O'Connor 1994; Wilson 2005).

In light of these worries, we suggest that compliance with Closure is a plausible further necessary condition for the truth of physicalism. In support of this we identify three jobs that Closure does for physicalists.

First, the failure to draw a modal distinction between physicalism and emergentism has not led philosophers to the conclusion that these positions are indistinguishable simpliciter. Instead, the debate between physicalists and emergentists concerns the existence of novel higher-level powers or forces. For example, McLaughlin argues that the characteristic feature of British emergentism was the commitment to configurational forces - i.e. 'fundamental forces that can be exerted only by certain types of configurations of particles, and not by any types of pairs of particles' $(1992,21)$ - which are exerted 'downwardly' from higher special science levels to the physical level (ibid., 22). The existence of such forces would violate Closure and physicalism. More recently, contemporary emergentist philosophers have 
rejected Closure either implicitly by positing novel causal powers for mental properties (e.g., O’Connor 1994) or explicitly by arguing against the principle (e.g., Lowe 2013, 168-9, Hendry 2010, 187-188). Hence, Closure plays a central role in distinguishing physicalism from anti-physicalist views such as emergentism.

Second, we observe that popular physicalist attempts to flesh out the relation between mental and physical properties incorporate Closure. ${ }^{19}$ Consider first functionalism about the mind which comes in two versions; what McLaughlin (2006) dubs role-functionalism and fillerfunctionalism. ${ }^{20}$ Both views hold that mental properties are associated with certain causalfunctional roles and that physical properties functionally realize mental properties by playing or occupying these roles. According to role-functionalism, a mental property $M$ is the (second-order) property of having some physical property or another that plays the $M$-role. According to filler-functionalism, a mental property $M$ just is the (first-order) property that plays the $M$-role. The distinction between role- and filler-functionalism is not so important for our purposes. What is important, however, is that on either functionalist account of mental properties, the physical effects associated with having $M$ are caused by the physical property (or properties) that play the $M$-role. It is in virtue of causing these effects (i.e. playing that particular role) that the physical property in question is a functional realizer of $M$. In other words, both role- and filler-functionalism comes with Closure as a built-in feature.

\footnotetext{
${ }^{19}$ Or to be more precise, they incorporate that Closure holds for physical effects putatively caused by mental properties. Obviously, reductive physicalists incorporate Closure in this way - if mental properties are physical then causation by mental properties is causation by physical properties.

${ }^{20}$ The distinction is well-known in the literature although sometimes goes under different names. E.g. Block distinguishes between functional state identity views (which is the role-functionalist view) and functional specification views (which is the filler-functionalist view) (Block 1980, 179).
} 
Another example from the literature is the so-called subset view of realization, according to which the causal powers of any mental property $M$ are a proper subset of the causal powers of some physical property that realizes $M$ (Wilson 1999; 2011; Shoemaker 2001; 2007). Again, we see that this view comes with Closure as a built-in feature: $M$ exercising a power $p$ to cause some physical effect guarantees that a physical property $N$ is exercising a power to cause the same effect since $p$ is a power of both $M$ and $N$. More generally, Wilson (2015) argues that all forms of physicalism must adhere to claim that the causal powers of mental properties are included among the causal powers of physical properties.

Third and finally, there are epistemic reasons for physicalists to endorse Closure. Firstly, Papineau (2001) traces the rise of physicalism in the last century to empirical evidence for Closure becoming available. The flipside of this is the downfall of emergentism which occurred in light of the same empirical evidence according to McLaughlin (1992). If so, rejecting Closure would undermine a key historical motivation for physicalism. Secondly, as noted above, psycho-physical supervenience is generally thought to be a necessary condition for physicalism. Physicalists therefore ought to have reasons for thinking that psychophysical supervenience holds. For instance, why does Jones' neural property $N$ necessitate $M$ rather than some other mental property? The best candidate for such an explanation is arguably a causal one. For instance, a role-functionalist can answer that $N$ necessitates $M$ because $N$ plays the causal role associated with $M$ rather than the causal role of some other mental property. Similarly, a proponent of the subset view can answer that $N$ correlates with $M$ because the powers of $N$ include the powers of $M$ as a subset. If Closure fails, such causal explanations will be unavailable. Hence, Closure plays an evidential role in supporting physicalism. 
We take these three observations to strongly suggest that Closure is a requirement of physicalism about the mind, at least as far as how physicalism is understood in the contemporary debate. The incompatibilists' rejection of Closure is therefore problematic from a physicalist viewpoint.

\subsection{Weak closure}

That the truth of Closure is a necessary condition for physicalism might strike you as an unsurprising claim. After all, most physicalist are happy to accept it. Why then do Menzies and List reject it while insisting that their response to the exclusion problem is a physicalist response? The reason, we believe, is that Menzies and List cash out the idea that the physical domain is causally closed using a weaker principle than Closure. Importantly, it is weak enough that their view is compatible with it. So there is an objection that we need to consider and defuse. The objection grants that physicalism requires the causal closure of the physical domain but holds that Closure, as we stated it above, presents an unnecessarily strong formulation of this idea. To recall, Closure was stated as follows:

(Closure) If a physical event $e$ has a cause at time $t$, then $e$ has a sufficient physical cause at $t$.

Menzies and List, instead, use the following claim to express that causal closure of the physical (note: they simply call this 'closure'):

(Weak Closure) If a physical event $e$ has a cause at time $t$, then there is a physical property at $t$ which is causally sufficient for $e^{21}$

\footnotetext{
${ }^{21}$ See List and Menzies $(2009,476)$. See also Menzies $(2008,198)$.
} 
Under difference-making accounts of causation, Weak Closure is weaker than Closure because being causally sufficient for $e$ is weaker than being a sufficient cause of $e$. Causal sufficiency here is understood as nomological sufficiency: $F$ at $\mathrm{t}_{1}$ is causally sufficient for $G$ at $\mathrm{t}_{2}$ if and only if it is nomologically necessary that $F$ at $\mathrm{t}_{1}$ will be followed by $G$ at $\mathrm{t}_{2}$ (Menzies 2013, 63; see also Woodward 2008, 228-229). ${ }^{22}$ Compared to causation, causal sufficiency is an indiscriminate concept. For instance, a man taking birth control pills is causally sufficient for him not getting pregnant but it is not (according to DM theorists) a cause of this. $^{23}$

Now, as we have seen, proportionalists deny that, in normal cases of mental causation, the physical realizer is a cause of the relevant behavioural effect. However, the physical realizer is nevertheless causally sufficient for the effect. This follows from the fact that the causally efficacious mental property supervenes on its realizer with at least nomological necessity. In other words, it is a matter of nomological necessity that when $N$ is instantiated, $B$ follows since $N$ nomologically necessitates a property that causes $B$. According to proportionalism, while normal cases of mental causation violate Closure, they therefore do not violate Weak Closure. Hence, if Weak Closure is good enough for physicalism under a proportionalist account of causation, our argument that the incompatibilist solution to the exclusion problem undermines physicalism can be deflected.

We respond that Weak Closure is not good enough for physicalism since it cannot do the jobs Closure does for physicalists that we identified above. First, the physicalism-emergentism

\footnotetext{
${ }^{22}$ Like Menzies, we assume determinism here; nothing substantial hinges on this assumption.

${ }^{23}$ We borrow this example from Menzies (2013), who in turn borrows it from Salmon (1971). We are assuming, with these authors, that the man in this example is biologically male and hence incapable of being pregnant.
} 
debate cannot be understood as a debate over the truth of Weak Closure since emergentism arguably entails Weak Closure: in cases of emergent causation, the physical emergence base is causally sufficient for the relevant physical effect since the base nomologically necessitates the causally efficacious emergent property. For the same reason, availability of evidence for Weak Closure does not explain the rise of physicalism and the downfall of emergentism.

Second, neither functionalism nor the subset view can be construed as incorporating merely Weak Closure. Functionalism (in both its role- and filler-functionalist guises) is the view that physical properties functionally realize mental properties because the former play the roles associated with the latter and to play a causal role is to cause the effects specified by that causal role. A similar point applies to the subset view's use of causal powers. A causal power is a power to cause some effect; it is not a power to be merely causally sufficient for some effect.

Third, we argued that physicalists can appeal to Closure in order to justify that psychophysical supervenience holds. Weak Closure, on the other hand, is not capable of justifying this supervenience relation. Cases of mental causation comply with Weak Closure in virtue of the fact that $M$ supervenes on $N$ with nomological necessity. So, the obtaining of psychophysical supervenience is not due to Weak Closure holding; it is the other way around. That is, it is by virtue of the fact that $N$ necessitates $M$ with nomological necessity that $N$ is causally sufficient for effects caused by $M$. Hence, Weak Closure cannot be used to explain or justify that very same supervenience relation.

In sum, we believe that physicalists cannot replace the commitment to Closure with a commitment to Weak Closure under a proportionalist account of causation. If so, the incompatibilist solution to the exclusion problem offered by Menzies and List is not available to physicalists. Insofar as incompatibilists can respond to the objection that their theory of 
causation requires disjunctive properties, which in turn threatens the non-reductionist component of non-reductive physicalism, they run into this further problem that their treatment of mental causation threatens the physicalist component of non-reductive physicalism.

\section{Conclusion}

We examined an incompatibilist solution to the exclusion problem. This solution is based on a proportionalist understanding of difference-making, and it rejects Closure. We argued that the incompatibilist solution is not attractive from a non-reductive physicalist point of view. This is because, from a non-reductive physicalist point of view, incompatibilism faces two problems. First, the proportionalist theory of causation that incompatibilism is based on requires disjunctive properties, and if disjunctive properties are allowed in one's ontology, then one's response to the disjunctive move against non-reductionism is undercut. Second, rejecting Closure is problematic from a physicalist perspective. We also noted that these two problems are mutually exclusive: incompatibilism leads to either reductive physicalism or non-physicalism, but not to both. We have stayed neutral as to which problem is more serious. Either way, incompatibilism is not available to non-reductive physicalism.

Acknowledgment: Many thanks to Lise Marie Andersen, Peter Fazekas, Neil McDonnell, Nathan Wildman and two anonymous referees for their helpful comments on previous versions of this paper.

\section{References}


Armstrong, D. M. 1989. Universals: An Opinionated Introduction. Westview Press.

Audi, P. 2013. "How to Rule Out Disjunctive Properties.” Noûs 47 (4):748-766.

Beebee, H. 2017. “Epiphenomenalism for Functionalists.” In H. Beebee et al. (eds.), Making a Difference: Essays on the Philosophy of Causation. Oxford: Oxford University Press.

Block, N. 1980. “Introduction: What is Functionalism?" In Ned Block (ed.): Readings in Philosophy of Psychology, Vol. One. Harvard University Press.

Clapp, L. 2001. “Disjunctive Properties: Multiple Realizations.” Journal of Philosophy 98 (3):111-136.

Fazekas, P. 2014. "Pursuing Natural Piety: Understanding Ontological Emergence and Distinguishing it from Physicalism.” Dialectica 68 (1):97-119.

Fodor, J. A. 1974. "Special sciences (or: The disunity of science as a working hypothesis)." Synthese 28 (2):97-115.

Franklin-Hall, L. R. 2016. "High-Level Explanation and the Interventionist's 'Variables Problem'.” British Journal for the Philosophy of Science 67 (2):553-577.

Heil, J. 1992. The Nature of True Minds. Cambridge University Press.

Hendry, R. F. 2010. “Ontological Reduction and Molecular Structure.” Studies in History and Philosophy of Modern Physics 41: 183-191.

Horgan, T. 1982. “Supervenience and microphysics.” Pacific Philosophical Quarterly 63 (January):29-43. 
Horgan, T. 1993. "From supervenience to superdupervenience: Meeting the demands of a material world." Mind 102 (408):555-86.

Horgan, T. 1997. “Kim on Mental Causation and Causal Exclusion.” Philosophical Perspectives 11.

Jackson, F. 1998. From Metaphysics to Ethics: A Defence of Conceptual Analysis. Oxford University Press.

Jaworski, W. 2002. "Multiple-realizability, explanation and the disjunctive move." Philosophical Studies 108 (3):289 - 308.

Kim, J. 1984. "Concepts of supervenience.” Philosophy and Phenomenological Research 45:153-76.

Kim, J. 1992. "Multiple realization and the metaphysics of reduction." Philosophy and Phenomenological Research 52 (1):1-26.

Kim, J. 1998. Mind in a Physical World: An Essay on the Mind-Body Problem and Mental Causation. MIT Press.

Kim, J. 2005. Physicalism, or Something Near Enough. Princeton University Press.

Lewis, D. 1983. “New Work for a Theory of Universals.” Australasian Journal of Philosophy 61 (December):343-377.

List, C. \& Menzies, P. 2009. "Nonreductive Physicalism and the Limits of the Exclusion Principle.” Journal of Philosophy 106 (9):475-502. 
Lowe, E. J. 2013. "Substance Causation, Powers, and Human Agency.” In S.C. Gibb et. al. (eds.) Mental Causation and Ontology. Oxford: Oxford University Press: 153-172.

McDonnel, N. 2016. "Causal Exclusion and the Limits of Proportionality." Philosophical Studies 174 (6):1-16.

McLaughlin, B. 1992. "The Rise and Fall of British Emergentism.” In A. Beckerman, et. al. (eds.), Emergence or Reduction? Essays on the Prospects of Non-Reductive Physicalism. Berlin: De Gruyter:49-93.

McLaughlin, B. 2006. "Is Role-functionalism Committed to Epiphenomenalism?” Journal of Consciousness Studies 13:39-66.

Menzies, P. \& List, C. 2010. “The Causal Autonomy of the Special Sciences.” In Cynthia Mcdonald \& Graham Mcdonald (eds.), Emergence in Mind. Oxford University Press.

Menzies, P. 2008. "The Exclusion Problem, the Determination Relation, and Contrastive Causation.” In Jakob Hohwy \& Jesper Kallestrup (eds.), Being Reduced: New Essays on Reduction, Explanation, and Causation. Oxford University Press.

Menzies, P. 2013. "Mental causation in the physical world." In Sophie C. Gibb \& Rögnvaldur Ingthorsson (eds.), Mental Causation and Ontology. Oxford University Press.

Noordhof, P. 2003. "Not Old ... but not that New either: Explicability, Emergence and the Characterisation of Materialism." In S. Walter \& H. Heckman (eds.), Physicalism and Mental Causation: The Metaphysics of Mind and Action. Exeter: Imprint Academic:85-108.

Noordhof, P. 2010. "Emergent Causation and Property Causation.” In C. Macdonald \& G. Macdonald (eds.), Emergence in Mind. Oxford, Oxford University Press:69-99. 
O’Connor, T. 1994. “Emergent Properties.” American Philosophical Quarterly 31:91-104.

Papineau, D. 2001. “The rise of physicalism.” In Carl Gillett \& Barry M. Loewer (eds.), Physicalism and its Discontents. Cambridge University Press.

Penczek, A. 1997. "Disjunctive properties and causal efficacy." Philosophical Studies 86: 203-219.

Putnam, H. 1967. "Psychological Predicates.” In W.H. Capitan \& D.D. Merrill (eds.), Art, Mind and Religion. Pittsburgh: University of Pittsburgh Press: 37-48.

Raatikainen, P. 2010. "Causation, exclusion, and the special sciences.” Erkenntnis 73 (3):349-363.

Salmon, W. 1971. Statistical Explanation \& Statistical Relevance. University of Pittsburgh Press.

Shapiro, L. \& Sober, E. 2012. “Against proportionality.” Analysis 72 (1):89-93.

Shoemaker, S. 2001. "Realization and Mental Causation.” In C. Gillett \&B. Loewer (eds.), Physicalism and its Discontents. Cambridge: Cambridge University Press: 74-98.

Shoemaker, S. 2007. Physical Realization. Oxford: Oxford University Press.

Skiles, A. 2016. "In defense of the disjunctive.” Inquiry 59: 471-487.

Van Cleve, J. 1990. “Emergence vs. Panpsychism: Magic or Mind Dust?” Philosophical Perspectives 4:215-226.

Weslake, B. 2013. “Disjunctive Explanations.” Unpublished manuscript. 
Wilson, J. 1999. "How Superduper does a Physicalist Supervenience Need to Be?” Philosophical Quarterly 49:33-52.

Wilson, J. 2005. "Supervenience-based Formulations of Physicalism." Noûs 39:426-459.

Wilson, J. 2011. "Non-Reductive Realization and the Powers-Based Subset Strategy.” The Monist 94:121-154.

Wilson, J. 2015. “Metaphysical Emergence: Weak and Strong.” In T. Bigaj and C. Wuthrich (eds.), Metaphysics in Contemporary Physics: Poznan Studies in the Philosophy of the Sciences and the Humanities. Leiden: Brill:345-402.

Woodward, J. 2003. Making Things Happen: A Theory of Causal Explanation. Oxford University Press.

Woodward, J. 2008. “Mental causation and neural mechanisms.” In Jakob Hohwy \& Jesper Kallestrup (eds.), Being Reduced: New Essays on Reduction, Explanation, and Causation. Oxford University Press.

Woodward, J. 2015. “Interventionism and Causal Exclusion.” Philosophy and Phenomenological Research 91 (2):303-347.

Yablo, S. 1992. “Mental causation.” Philosophical Review 101 (2):245-280.77

Yablo, S. 1997. "Wide Causation.” Philosophical Perspectives 11. 